\title{
LA-UR-12-25060
}

Approved for public release; distribution is unlimited.

Title:

\section{Uncertainty Quantification with Monte Carlo Hauser-Feshbach Calculations of Prompt Fission Neutrons and Gamma Rays}

Author(s):

\author{
Talou, Patrick \\ Becker, Bjorn \\ Danon, Yaron \\ Kawano, Toshihiko \\ Stetcu, lonel
}

Intended for: $\quad$ Report

\section{- LosAlamos NATIONAL LABORATORY

\author{
EST. 1943
}

Disclaimer:

Los Alamos National Laboratory, an affirmative action/equal opportunity employer,is operated by the Los Alamos National

Security, LLC for the National NuclearSecurity Administration of the U.S. Department of Energy under contract DE-AC52-06NA25396.

By approving this article, the publisher recognizes that the U.S. Government retains nonexclusive, royalty-free license to

publish or reproduce the published form of this contribution, or to allow others to do so, for U.S. Government purposes.

Los Alamos National Laboratory requests that the publisher identify this article as work performed under the auspices of the

U.S. Departmentof Energy. Los Alamos National Laboratory strongly supports academic freedom and a researcher's right to publish; as an institution, however, the Laboratory does not endorse the viewpoint of a publication or guarantee its technical correctness. 


\title{
Uncertainty Quantification with Monte Carlo Hauser-Feshbach Calculations of Prompt Fission Neutrons and Gamma Rays
}

\author{
${ }^{1}$ P. Talou, ${ }^{2}$ B. Becker, ${ }^{2}$ Y. Danon, \\ ${ }^{1} \mathrm{~T}$. Kawano, and ${ }^{1} \mathrm{I}$. Stetcu \\ ${ }^{1}$ T-2 Nuclear Theory Group, Los Alamos National Laboratory, \\ Los Alamos, NM 87545, USA \\ ${ }^{2}$ Gaerttner LinaC Laboratory, Rensselaer Polytechnic Institute, \\ NY 12180, USA
}

September 27, 2012

\begin{abstract}
We have performed sensitivity calculations using the CGMF code that implements the statistical Hauser-Feshbach equations in a Monte Carlo fashion to describe the de-excitation of fission fragments by emission of prompt neutrons and gamma rays. The specific importance of several input quantities is emphasized. An uncertainty quantification method, considering sources of uncertainty from both model calculations and experiments, is outlined.
\end{abstract}

\section{Monte Carlo Hauser-Feshbach Calculations}

As reported in a separate document [1], we have developed a new code, CGMF , implementing the equations of the statistical Hauser-Feshbach reaction theory in a Monte Carlo fashion. The Monte Carlo procedure allows to more exactly and directly access exclusive quantities for the prompt neutrons and gamma rays, such as: $\mathrm{P}(\nu), \bar{\nu}(A, Z, T K E), \chi_{\nu=\nu_{0}}, \ldots$ (and similar quantities for the gamma rays). Obviously, one can also infer averaged quantities such as the average prompt fission neutron spectrum (PFNS) and average

*Present address: EC-JRC-IRMM, Neutron Physics Unit, Retieseweg 111, 2440 Geel, Belgium 
prompt fission neutron multiplicity (PFNM), which have been traditionally calculated in the Los Alamos or Madland-Nix model [2]. This model has been widely used to evaluate PFNS for a wide range of actinides, and for incident neutron energies from thermal up to 20 or $30 \mathrm{MeV}$. Several improvements have been implemented over the years [3, 4, 5], leading to better fits to measured PFNS. However, the LA model is limited in its scope to the prediction of average PFNS and PFNM, and does not say anything about prompt gamma rays.

\subsection{Model Input Parameters}

While CGMF can predict many more data than the Los Alamos model, it also has many more parameters to deal with. Most of these parameters have been evaluated independently of any spectrum or multiplicity measurement and can therefore be considered as fixed parameters, which are not to be easily tampered with in CGMF calculations. Those fixed parameters deal with specific characteristics of the fission fragments that are populated: nuclear masses, nuclear structure (discrete levels and continuum level density), etc. However, we should remember that for many fission fragments, which are neutron rich and away from the valley of stability, these quantities are only poorly known and model parameter systematics are often used to describe their nuclear structure beyond the few experimentally known levels.

Other parameters are related to the initial conditions of the Monte Carlo decay sequences. In the original Los Alamos model, only a few fission fragments near the peak of the distribution are considered, and no explicit information on the specific configurations (e.g., deformation) is required to perform the calculations. On the contrary, CGMF calculations are performed for all fission fragments characterized by mass $A$ and charge $Z$, but also by their specific initial excitation energy, spin and parity distribution.

Despite some recent progress [6], theoretical predictions for the fission fragment yields $Y(A, Z, U, J, \pi)$ are still missing, and one has to rely on experimental values and theoretical assumptions. For the reactions considered so far, we have reconstructed the $Y(A, Z, T K E)$ yields, where $T K E$ is the total kinetic energy and roughly represents the degree of quadrupole deformations of the fragments near scission, from partial distributions $Y(A)$, $Y(Z), Y(T K E)$ that have been obtained experimentally. Obviously, such reconstructed yields carry some uncertainties, which should be propagated through CGMF calculations.

For known fission fragment yields $Y(A, Z, T K E)$, the total excitation energy $T X E$ available for a specific fission pair can be inferred. All the 
energy will be released through the emission of neutrons and gamma rays from both light and heavy fragments. What is not known however is how $T X E$ is shared between the two fragments at scission. Experimental data that are fragment mass-dependent can be used to constrain the average initial excitation energies in both fragments. This energy sorting problem carries another source of uncertainties.

Finally, since Hauser-Feshbach calculations are performed, initial spin and parity populations in both fragments are needed. Very little is known experimentally or even theoretically about this question, and one has to test several hypotheses against experimental prompt fission gamma-ray data. Because most gamma rays are emitted following the neutron emission, the exact spin and parity population do not impact much the prompt fission neutron results, but of course do impact the results for the prompt gamma rays.

In the following, we study those different sources of uncertainties and check their impact on several physical quantities of interest.

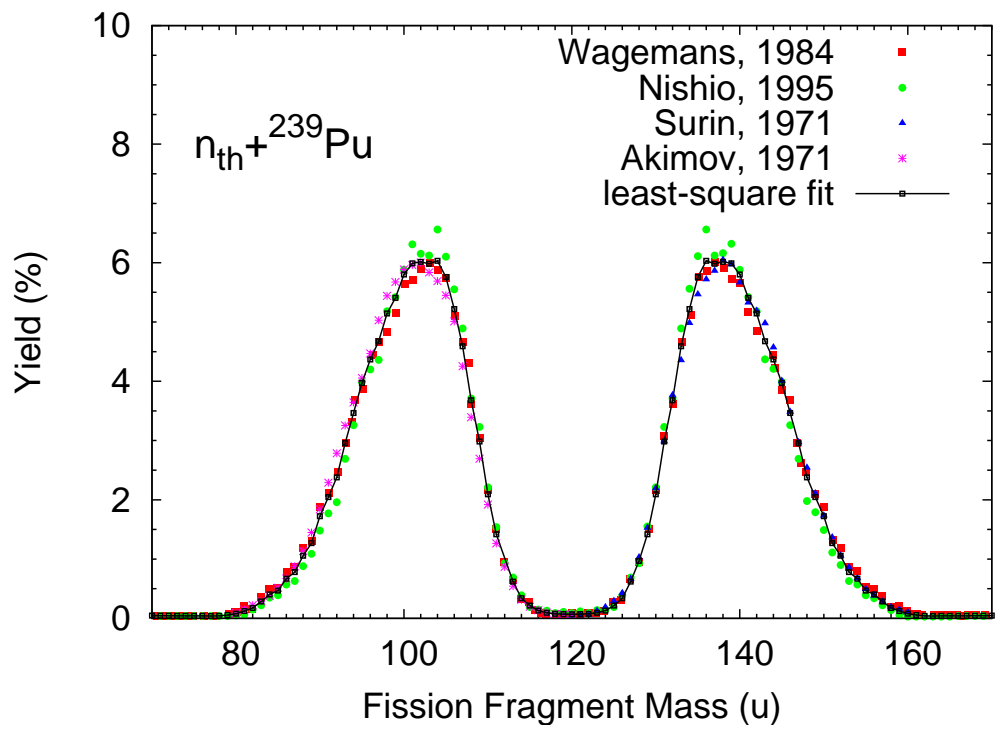

Figure 1: Primary fission fragment mass yields for thermal neutron-induced fission of ${ }^{239} \mathrm{Pu}$. 


\subsection{Fission Fragment Yields}

As explained at some length in [7], the pre-neutron emission fission fragment yields produced in the thermal neutron-induced fission reaction of ${ }^{239} \mathrm{Pu}$ were reconstructed from several measured quantities and systematics. The mass yields $Y(A)$ were obtained from a least-square fit of 4 experimental data sets, as shown in Fig. 1. In addition to differences observed between different experimental data sets, an additional uncertainty stems from the fact that the observed quantities are the fission products, i.e., after neutron emission, and have to be corrected using "known" average neutron multiplicity $\bar{\nu}(A)$. This quantity is unfortunately only known for very few isotopes and reactions, rendering such corrections difficult and uncertain.

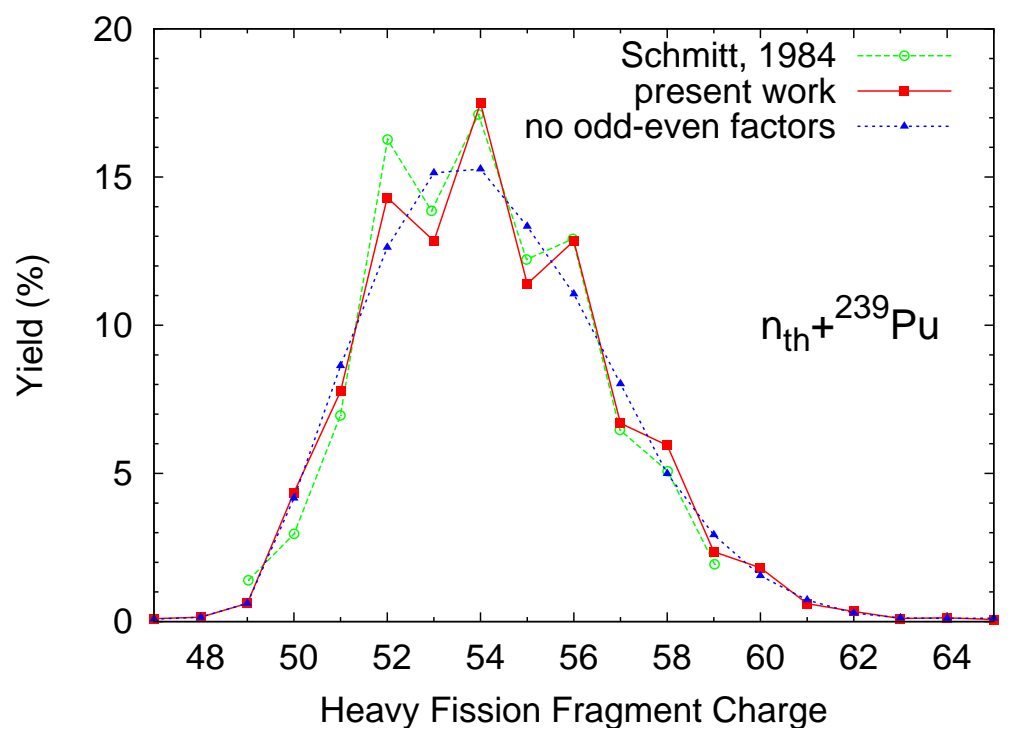

Figure 2: The fission fragment charge distribution (red) is shown as a function of the heavy fragment charge for the thermal neutron-induced fission of ${ }^{239} \mathrm{Pu}$. The results are shown in dotted blue if odd-even factors are neglected. The experimental data of Schmitt et al. [9] are also shown for comparison.

The conditional probabilities $Y(Z \mid A)$ were calculated with Wahl systematics [8]. Figure 2 shows the fission fragment yields as a function of the heavy fission fragment charge, inferred from the fully reconstructed yields $Y(A, Z)$, and compared to experimental data by Schmitt et al. [9]. The agreement between experimental data and reconstructed yields is quite good, but not 
perfect. The role played by odd-even effects is shown to be quite important in getting reasonable values. Uncertainties in the initial charge of the fragments are important as charges are not modified during the neutron and gamma emission cascade.
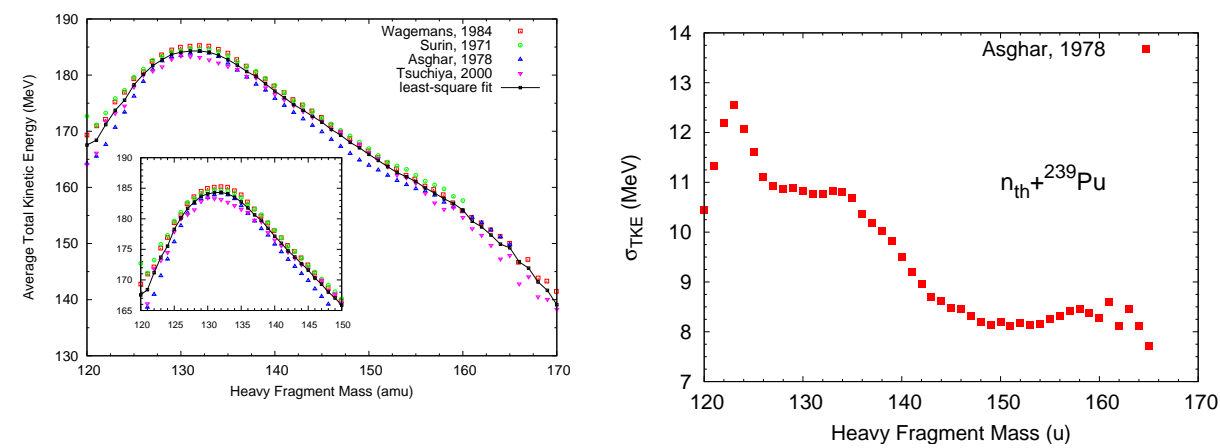

Figure 3: Fission fragment mass-dependent (a) average total kinetic energy $\langle T K E\rangle(A)$ and (b) distribution width $\sigma_{T K E}(A)$ as a function of the heavy fragment mass.

Another very important quantity is the total kinetic energy distribution $Y(T K E \mid A, Z)$. Again, it can be reconstructed from partial experimental information on $\langle T K E\rangle(A)$ and $\sigma_{T K E}(A)$, assuming that $Y(T K E \mid A)$ has a gaussian shape. These quantities are shown in Fig. 3. An accurate knowledge of $T K E$ is crucial as it directly impacts how much excitation energy is available for the emission of neutrons and gamma rays. As can be seen from this figure, the agreement (spread) between different experimental data sets is at the level of $1-3 \%$, peaking in the symmetric region at up to $5 \%$ discrepancy. A difference of $1 \%$ on TKE values near $170 \mathrm{MeV}$ translates into about $3.5 \mathrm{MeV}$ excitation energy difference, roughly half of what is needed for the emission of one neutron. This difference is fairly big and could have a large impact on CGMF predictions.

\subsection{Energy Sorting Mechanisms}

Once the initial yields in mass, charge and total kinetic energy are known, a couple of important questions remain: what is the excitation energy in each fragment, and what is their spin distribution? While the total excitation energy available for scission is known for a specific fission fragment pair given a particular $T K E$, the decomposition of this energy into intrinsic and 
collective terms, as well as between each fragment remains uncertain.

To model the energy partitioning at scission, we introduce a parameter $R_{T}$ defined as a ratio of the temperature in the light fragment $T_{l}$ to the temperature in its heavy partner $T_{h}$. There are strong experimental evidences that indicate that $T_{l}>T_{h}$ by the time the fragments are fully accelerated and evaporating neutrons. One of these evidences is the average neutron multiplicities of the light and heavy fragments. In all reactions studied so far $\left(\mathrm{n}_{t h}+{ }^{235} \mathrm{U}, \mathrm{n}_{t h}+{ }^{239} \mathrm{Pu},{ }^{252} \mathrm{Cf} s f, \ldots\right)$, experimental data show $\bar{\nu}_{l}>\bar{\nu}_{h}$.

The more detailed observation of the well-known saw-tooth for $\bar{\nu}(A)$ clearly indicates the important role of deformation and shell effects. The sorting of the intrinsic excitation energy is also important and has seen some new interesting developments [10]. However a clear and quantitative picture of the energy partitioning is still missing. In this work, we have been using $R_{T}(A)$ as a free parameter, which also depends on the specific mass split. This parameter is crucial and impacts significantly all quantities related to the neutron emission, except the fully average quantities such as the total multiplicity $\bar{\nu}_{\text {tot }}$ and spectrum $\langle\chi\rangle$. The average spectrum $\langle\chi\rangle$ is actually still impacted by a change in $R_{T}$ as the average spectra from light and heavy fragments have different hardnesses.

\section{Sensitivity Calculations}

We have studied the sensitivity of the results to the choice of model input parameters, starting with the fission fragment yields. As stated earlier, one has to rely on experimental data for this quantity, and therefore deal with inherent experimental uncertainties due to mass and energy resolutions, detector response uncertainties, and model-dependent errors. Indeed, what is often measured are not the primary fission fragment yields, but the postneutron emission fission product yields instead. To infer the primary fragment yields, one has to rely on our relatively poor knowledge of $\bar{\nu}(A, T K E)$, which is only known to some accuracy for a few isotopes and incident neutron energies. Assumptions are also made along the way on the particular neutron emission mechanisms, excluding the possibility of scission neutrons for instance.

For this study, we have relied on our earlier Monte Carlo evaporation code, FFD, which is very similar to CGMF in principle, but neglects the neutron-gamma competition by emitting neutrons from a Weisskopf spectrum instead of performing full Hauser-Feshbach calculations. We expect that these sensitivity studies would lead to the same qualitative results for 
the neutrons when using CGMF instead. Taking into account uncertainties in the initial fission fragment yields lead to a significant increase in the spread of the calculated distribution of $\bar{\nu}$ as can be seen in Fig. 4. In fact, a large fraction of this uncertainty comes from uncertainties in the total kinetic energy values.

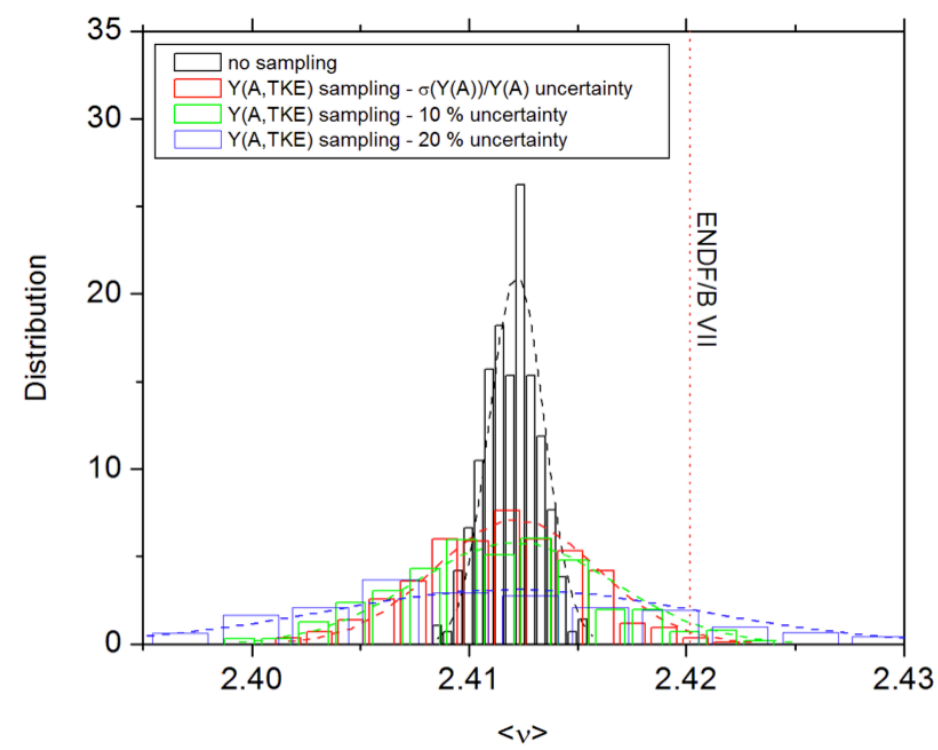

Figure 4: The calculated distributions of average prompt fission neutron multiplicity $\bar{\nu}$ for the thermal neutron-induced fission reaction on ${ }^{235} \mathrm{U}$ are shown assuming different uncertainties in the fission fragment yields used. In this work, the FFD evaporation code was used instead of CGMF, but we expect the results to be qualitatively equivalent when using the more sophisticated Hauser-Feshbach calculations.

We have also studied the impact of the Pulse Height Defect (PHD) correction used in the data reduction. This parameter can lead to a correlated shift in the yield matrix $Y(A, T K E)$ mainly in terms of $T K E$. By randomly sampling an offset of this parameter from a Gaussian distribution with the width of 0.5 or $1.0 \mathrm{MeV}$, a significant uncertainty on the final neutron multiplicity is introduced (see results in Table 1). An impact on the uncertainty of $\bar{\nu}(A)$ and on the uncertainty of the average spectrum was observed as well.

In Monte Carlo Hauser-Feshbach calculations, the neutron separation energies $S_{n}$ for all fragments that play part in the evaporation cascades are 
Table 1: Uncertainty in calculated neutron multiplicities due to Pulse Height Defect (PHD) corrections.

\begin{tabular}{|c|c|c|c|}
\hline & $\nu_{\text {tot }}$ & $\nu_{h}$ & $\nu_{l}$ \\
\hline No Sampling & $2.4323 \pm 0.0009$ & $1.3739 \pm 0.0009$ & $1.0589 \pm 0.0007$ \\
$\Delta_{P H D}=0.5 \mathrm{MeV}$ & $2.4372 \pm 0.1132$ & $1.3770 \pm 0.0674$ & $1.0602 \pm 0.0458$ \\
$\Delta_{P H D}=1 \mathrm{MeV}$ & $2.4412 \pm 0.2302$ & $1.3803 \pm 0.1374$ & $1.0610 \pm 0.0928$ \\
\hline
\end{tabular}

used to compute the residual excitation energies in the daughter fragments. The values for $S_{n}(A, Z)$ are taken from the 2003 Audi et al. evaluated values [11], in which standard deviations were evaluated for every nuclide. We have performed many FFD calculations using sampled values for $S_{n}(A, Z)$. Interestingly, evaluated uncertainties in $S_{n}$ values led to an increase of the calculated uncertainty on $\bar{\nu}$ by a factor of two.

Moving from experimental uncertainties to model parameter uncertainties, we now turn our attention to an important parameter in CGMF calculations that is the ratio $R_{T}$ of initial temperatures in the light and heavy fragments. Modifying $R_{T}$ has a strong impact on prompt fission neutron characteristics, including spectrum, multiplicity distribution, etc. It has only a limited impact on the average total neutron multiplicity $\bar{\nu}$ as it does not change the available total excitation energy in the system. However, it does modify the number of neutrons that are emitted from the light vs. heavy fragment. The light fragment tends to have a harder spectrum than the heavy one, therefore changing $R_{T}$ will also change the spectrum.

The impact of $R_{T}$ on the prompt neutron multiplicity distribution $\mathrm{P}(\nu)$ is shown in Fig. 5 for the thermal neutron-induced fission of ${ }^{239} \mathrm{Pu}$. In this case, the changes are modest, but still visible.

As expected, modifying the excitation energy partitioning between the two fragments has a significant impact on mass-dependent neutron results, such as $\bar{\nu}(A)$ shown in Fig. 6a for the thermal neutron-induced fission of ${ }^{235} \mathrm{U}$. On the contrary, this particular choice for this parameter has very little impact on prompt $\gamma$-ray characteristics, since those are mostly emitted following neutron emission. The average prompt $\gamma$-ray multiplicity $\bar{N}_{\gamma}(A)$ is shown in Fig. 6b,

We have also studied the impact of the initial spin distributions in the fission fragments on the calculated average prompt gamma-ray spectrum $\left\langle\chi\left(E_{\gamma}\right)\right\rangle$. The results are illustrated in Fig. 7 for the thermal neutron-induced fission of ${ }^{235} \mathrm{U}$ for different values of the $\alpha_{I}$ parameter that is used to increase 


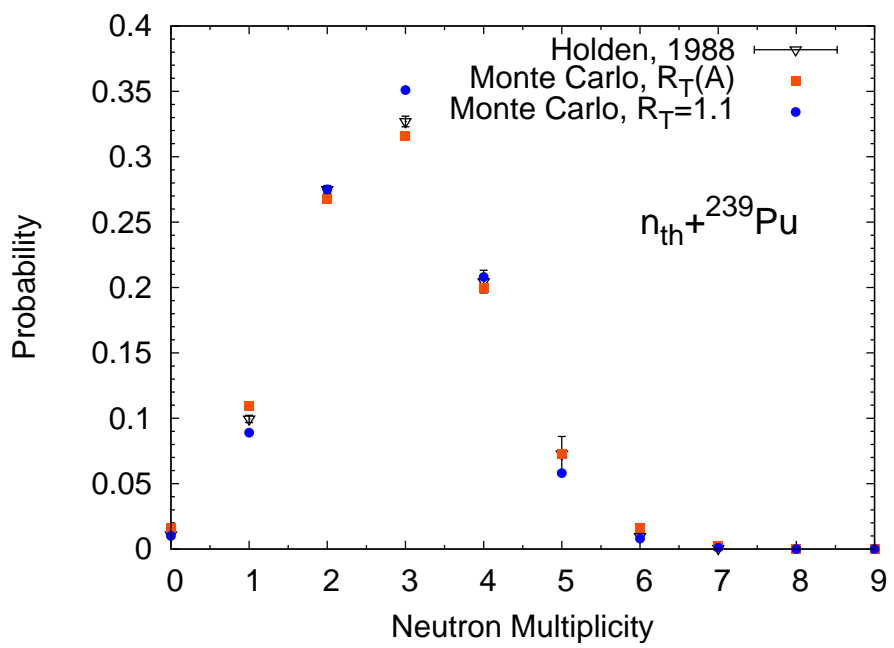

Figure 5: Prompt fission neutron multiplicity distribution $\mathrm{P}(\nu)$ calculated for the $\mathrm{n}_{t h}+{ }^{239} \mathrm{Pu}$ fission reaction, for different values of the $R_{T}$ parameter.

$\left(\alpha_{I}>1\right)$ or decrease $\left(\alpha_{I}<1\right)$ the average initial spin. Increasing $\alpha_{I}$ leads to a softer prompt gamma-ray spectrum with a larger gamma-ray multiplicity.

\section{Combining Experiments and Model Calculations}

The last section showed the sensitivity of the CGMF results to the choice of model input parameters. In order to evaluate uncertainties stemming from this approach, experimental data have to be included in the analysis to constrain the phase-space explored by the model parameters. In previous works $[12,13,14]$, the Kalman filtering technique [15] has been used successfully to produce most of the covariance matrices currently in the ENDF/B-VII.1 library, in the fast neutron energy range. It uses a Bayesian updating technique, and in first order, the response of the calculated quantities to model parameter values is assumed to be linear. Model sensitivity calculations are performed and folded with experimental data covariance matrices to obtain posterior values for the parameters and the calculated physical quantities.

This procedure works fine when the number of parameters is small, and that the response is indeed almost linear, however it becomes very cumbersome when the number of parameters is very large, and inadequate when the response is not linear. An alternative technique is to sample distributions 

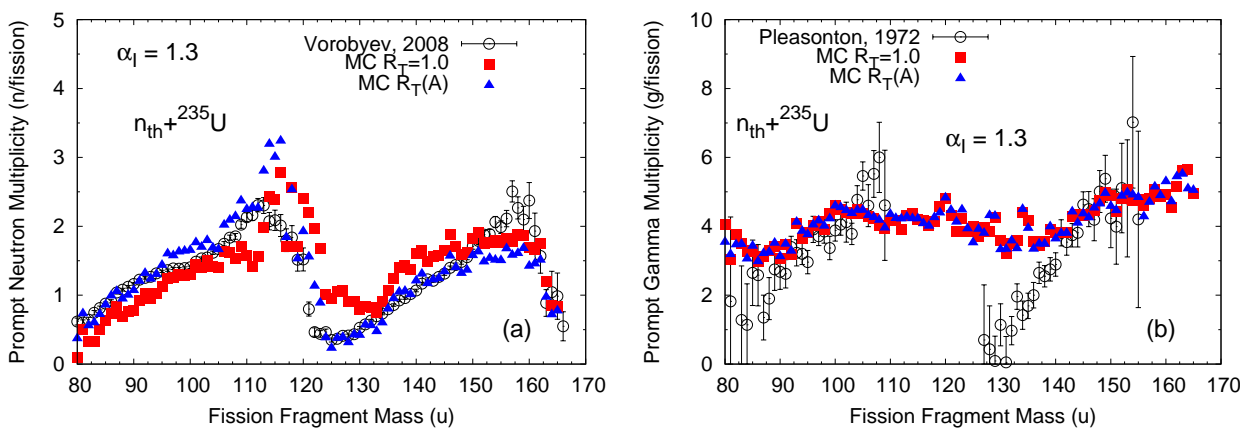

Figure 6: Calculated (a) $\bar{\nu}(A)$ and (b) $\bar{N}_{\gamma}(A)$ are shown for two different treatments of the $R_{T}$ parameter. Modifying $R_{T}(A)$ has a significant impact on the calculated average neutron multiplicities, but little effect on the gamma-ray average multiplicities.

with the Monte Carlo technique and perform a least-square minimization of the calculated results versus experimental data. Such a technique is the Unified Monte Carlo (UMC) approach exemplified with a toy model by Capote and Smith [16], and explored in more depth for an actual prompt fission neutron spectrum evaluation [17].

We plan to use the UMC approach to evaluate covariance matrices for prompt fission neutron data that are predicted through Monte Carlo HauserFeshbach calculations. Because CGMF can calculate much more than an average spectrum and multiplicity, other types of data, e.g., $\mathrm{P}(\nu), \bar{\nu}(A, T K E)$, $\mathrm{n}-\mathrm{n}$ energy and angular correlations, etc, can be used in the minimization procedure.

\section{Conclusion}

Using our CGMF code, we have identified the important parameters that impact Monte Carlo Hauser-Feshbach calculations. In particular, we have separated model input parameters into two categories: (1) the initial conditions for the fission fragments given experimentally as $Y(A), Y(Z), Y(T K E)$, $Y(A, T K E)$, etc; (2) parameters that define the initial energy, spin and parity distributions in each fission fragment before decay. In the first category, uncertainties are driven by experimental resolutions, detector limitations, etc. In the second category, our limited theoretical knowledge, and to some extend, comparison with post-scission data help constrain the distribution spreads. 


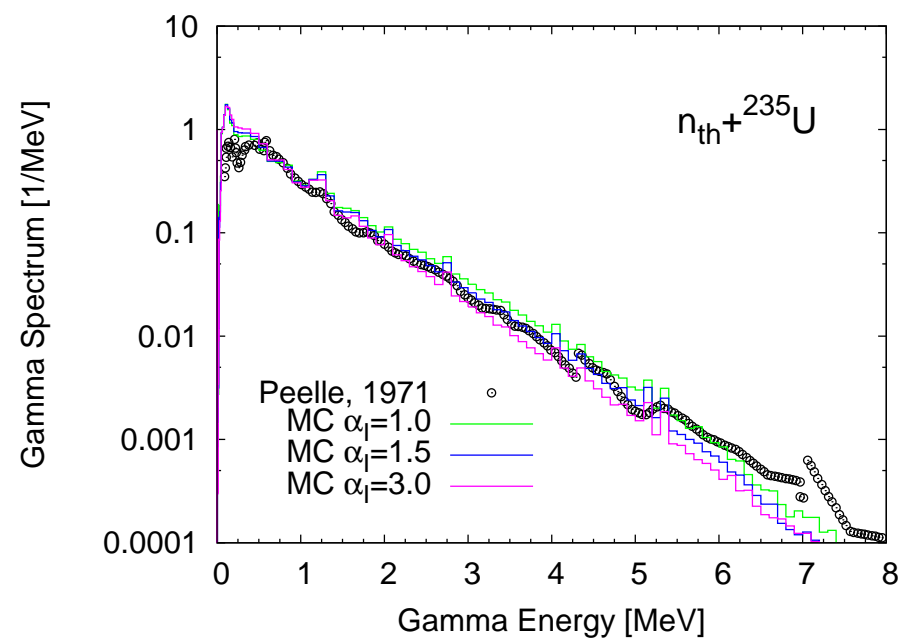

Figure 7: Prompt fission gamma-ray spectrum calculated in the $\left(n_{t h}, f\right)$ reaction on ${ }^{235} \mathrm{U}$, for three different values of the parameter $\alpha_{I}$. Increasing $\alpha_{I}$ leads to an increase in the average initial spin of the fragments, which in turns leads to a higher probability of emission of gamma rays at the expense of the prompt neutrons.

Numerical results showing the impact of those parameters on the final results have been reported. The important roles played by the total kinetic energy distribution and the specific energy sorting mechanisms near scission have been emphasized.

A natural extension of this work is to evaluate covariance matrices for actual prompt fission neutron data by folding model calculations with experimental data uncertainties. The Unified Monte Carlo approach appears to be a good candidate for this type of uncertainty quantification.

** This report represents the completion of our Level3 Milestone M3FT12LA0210023.

\section{Acknowledgements}

This work was supported in part by the AFCI Nuclear Energy University Program, grant award DE-AC07-05ID14517 sub contract 00091100. It was performed in part under the auspices of the National Nuclear Security Administration of the U.S. Department of Energy at Los Alamos National 
Laboratory under Contract No. DE-AC52-06NA25396.

\section{References}

[1] P. Talou, T. Kawano and I. Stetcu, "Monte Carlo Hauser-Feshbach Calculations of Prompt Fission Neutrons and Gamma Rays: Application to Thermal Neutron-Induced Fission Reactions on ${ }^{235} \mathrm{U}$ and ${ }^{239} \mathrm{Pu}$," Report to AFC, Milestone M3FT-12LA0210026 (2012).

[2] D.G. Madland and J.R. Nix, Nucl. Sci. Eng. 81, 213 (1982).

[3] G. Vladuca and A. Tudora, Ann. Nucl. Energy 28, 419 (2001).

[4] A. Tudora, Ann. Nucl. Energy 36, 72 (2009).

[5] T. Ohsawa, T. Horiguchi, M. Mitsuhashi, Nucl. Phys. A665, 3 (2000).

[6] J. Randrup and P. Möller, Phys. Rev. Lett. 106, 132503 (2011).

[7] P. Talou, B. Becker, T. Kawano, M.B. Chadwick, and Y. Danon, Phys. Rev. C 83, 064612 (2011).

[8] A.C. Wahl, LANL Report LA-13928 (2002).

[9] C. Schmitt, A. Guessous, J.P. Bocquet, H.-G. Clerc, R. Brissot, D. Engelhardt, H.R. Faust, F. Gönnenwein, M. Mutterer, H. Nifenecker, J. Pannicke, C. Ristori, and J.P. Theobald, Nucl. Phys. A430, 21 (1984).

[10] K.-H. Schmidt and B. Jurado, Phys. Rev. Lett. 104, 212501 (2010).

[11] G. Audi, A.H. Wapstra, and C. Thibault, Nucl. Phys. A729, 337 (2003).

[12] P. Talou, T. Kawano, D.G. Madland, A.C. Kahler, D.K. Parsons, M.C. White, R.C. Little, and M.B. Chadwick, Nucl. Sci. Eng. 166, 254 (2010).

[13] P. Talou, P.G. Young, T. Kawano, M. Rising and M.B. Chadwick, Nuclear Data Sheets 112, 3054 (2011).

[14] M.E. Rising, P. Talou, T. Kawano, and A.K. Prinja, Los Alamos Report LA-UR-12-21035, submitted to Nucl. Sci. Eng. 
[15] R.E. Kalman, Trans. ASME J. Basic Eng. 82 (D), 35 (1960).

[16] R. Capote and D.L. Smith, Nuclear Data Sheets 109, 2768 (2008).

[17] M.E. Rising, PhD Dissertation, University of New Mexico, to be published. 